\title{
The ability of positron emission tomography/computed tomography to detect synchronous colonic cancers in patients with obstructive colorectal cancer
}

\author{
CHIYO MAEDA, SHUNGO ENDO, YUICHI MORI, SHUMPEI MUKAI, \\ EIJI HIDAKA, FUMIO ISHIDA and SHIN-EI KUDO
}

Digestive Disease Center, Showa University Northern Yokohama Hospital, Yokohama, Kanagawa 224-8503, Japan

Received August 17, 2018; Accepted December 13, 2018

DOI: $10.3892 /$ mco.2019.1815

\begin{abstract}
Positron emission tomography/computed tomography (PET/CT) is frequently used to detect colorectal cancer. The present retrospective study assessed the ability of PET/CT to identify synchronous colonic lesions in 72 patients with obstructive colorectal cancer. All patients had undergone surgical resection without undergoing preoperative total colonoscopy (TCS) at the Digestive Disease Center (April 2007 to September 2016), and subsequently underwent TCS of the proximal colon within 2 years post-surgery. A total of 11 patients exhibited 18F-fluorodeoxyglucose uptake during $\mathrm{PET} / \mathrm{CT}$ of the proximal colon (4 invasive cancers, 3 advanced adenomas and 4 false-positive results), and 61 patients had no uptake in the proximal colon. Among these 61 patients, postoperative TCS revealed 2 invasive cancers and 4 advanced adenomas. The sensitivity of PET/CT for detecting synchronous invasive cancers was $66.6 \%(4 / 6)$, with a specificity of $89.4 \%$ (59/66), a positive predictive value of $36.4 \%$ (4/11), a negative predictive value of $96.7 \%(59 / 61)$, and an accuracy of $87.5 \%$ (63/72). Negative PET/CT results indicated a low probability of synchronous lesions in the proximal colon. Thus, PET/CT may be a useful tool for detecting synchronous colonic cancers in patients with obstructive colon cancer.
\end{abstract}

Correspondence to: Dr Chiyo Maeda, Digestive Disease Center, Showa University Northern Yokohama Hospital, Chigasak-Chuo 35-1, Tsuzuki-ku, Yokohama, Kanagawa 224-8503, Japan

E-mail: maechiyo@hotmail.co.jp

Abbreviations: $\mathrm{PET} / \mathrm{CT}$, positron emission tomography/computed tomography; TCS, total colonoscopy; FDG, fluorodeoxyglucose; SUV, standardized uptake value

Key words: PET/CT, synchronous colonic cancers, obstructive colorectal cancer

\section{Introduction}

Positron emission tomography/computed tomography $(\mathrm{PET} / \mathrm{CT})$ is sequential $\mathrm{CT}$ and PET scans that are performed during a single procedure using a hybrid PET/CT scanner. This modality combines the advantages of PET's high contrast resolution and CT's high spatial resolution. Furthermore, intravenous contrast agents are not required for PET/CT, because glucose metabolism is visualized using fluorodeoxyglucose (FDG) uptake, which is quantified and evaluated based on the standardized uptake value (SUV). As glucose metabolism is enhanced in colon cancer cells (1), $\mathrm{PET} / \mathrm{CT}$ is useful for detecting primary colon cancer, metastatic lesions, and recurrent lesions $(1,2)$.

Synchronous colon cancers are defined as two or more distinct primary tumors that are separated by normal mucosa, which excludes direct extension or metastasis of another lesion (3). Synchronous tumors are discovered within one year after surgery (4), and occur in 2-5\% of patients with colorectal cancer $(5,6)$. Although total colonoscopy (TCS) is considered the most sensitive and specific method for identifying colorectal lesions $(7,8)$, it cannot be performed for patients with an obstructive colorectal cancer. Thus, the American Society of Clinical Oncology and the American Cancer Society recommend follow-up TCS within 6 months after resection for patients with an obstructive cancer $(9,10)$. Patients who cannot undergo preoperative TCS may be evaluated for proximal lesions using barium enema, CT colonography, intraoperative palpation, or intraoperative colonoscopy. However, magnetic resonance imaging and CT are not ideal for detecting small lesions, and the inability of barium or air to pass through the obstruction may limit the use of CT colonography or barium enema. Furthermore, the use of barium can increase the risk of barium inspissation, which may delay adequate cleansing of the colon before surgery (11), and $\geq 40 \%$ of lesions around the splenic flexure can be missed during intraoperative palpation (12). Moreover, intraoperative colonoscopy can be limited by the patient's physical position and expansion of the intestinal tract by air from the endoscope. As $\mathrm{PET} / \mathrm{CT}$ is less invasive, compared to the previously mentioned modalities, the present study aimed to evaluate the ability of $\mathrm{PET} / \mathrm{CT}$ to detect proximal synchronous colonic cancers in patients with obstructive colorectal cancer. 


\section{Materials and methods}

Patients. The present study's retrospective protocol was approved by the Ethics Committee of Showa University Northern Yokohama Hospital (1310-14). Between January 2007 and September 2016, 2,796 patients with colorectal cancer underwent surgical resection at the Showa University Northern Yokohama Hospital. Among these individuals, 417 patients with obstructive colorectal cancer could not undergo preoperative TCS. The exclusion criteria were patients who underwent right hemicolectomy and ileocecal resection (complete resection of the proximal colon), and patients who had familial adenomatous polyposis, ulcerative colitis, or Crohn's disease. A total of 275 eligible patients required evaluation of the proximal colon, and 180 of these patients also underwent TCS within 2 years after surgery. These 180 patients included 72 patients who underwent both preoperative PET/CT and postoperative TCS, and 108 patients who underwent only postoperative TCS (Fig. 1).

Methods. For the present study, we defined 'advanced adenoma' as adenomas with a diameter of $>10 \mathrm{~mm}$ and high-grade dysplasia adenoma/mucosal cancer. 'Advanced lesions' were defined as invasive cancers and advanced adenomas. 'Obstructive colorectal cancer' was defined as cases in which the colonoscope could not reach the cecum because of cancer-related obstruction or stenosis.

The PET/CT was performed after the patients completed a fast of $\geq 4 \mathrm{~h}$, and their blood glucose levels were measured before the FDG administration to ensure that they were within the normal range. All patients received $185 \mathrm{MBq}$ of FDG, and the uptake period was defined as $60 \mathrm{~min}$. The PET/CT was performed using a Siemens Biograph 16 (Siemens Healthcare, Berlin, Germany), with the following imaging parameters: slice thickness of $1.5 \mathrm{~mm}$, total scale time of $30 \mathrm{sec}$, tube current-time of $50 \mathrm{mAs}$, and tube potential of $120 \mathrm{kV}$. The SUV was defined as the concentration of FDG in the tissue or lesion of interest, and was calculated as $\mathrm{SUV}=$ concentration $[\mathrm{MBq} / \mathrm{g}] /$ /injected dose $[\mathrm{MBq}]$ / patient's body weight $[\mathrm{g}])$. The SUVmax was defined as the maximum SUV value in a region of interest (13), and we defined malignancy as focal FDG uptake above the level of the surrounding tissue with an SUV of $>3.5$ (1).

\section{Results}

The association between FDG uptake and proximal colonic lesions. All 72 patients who underwent preoperative PET/CT plus postoperative TCS within 2 years exhibited FDG uptake in the main tumor, and the mean SUVmax in the main tumor was 15.0 (range: 9.3-24.7). The association between FDG uptake and proximal colonic lesions detected using postoperative TCS is shown in Table I. The sensitivity of PET/CT for detecting synchronous colon lesions was $53.8 \%$ (7/13), with a specificity of $93.2 \%(55 / 59)$, a positive predictive value of $63.6 \%$ (7/11), a negative predictive value of $90.2 \%$ (55/61), and an accuracy of $86.1 \%$ (62/72). The sensitivity of PET/CT for detecting synchronous invasive cancers was $66.6 \%(4 / 6)$, with a specificity of $89.4 \%(59 / 66)$, a positive predictive value of $36.4 \%$ (4/11), a negative predictive value of $96.7 \%$ (59/61), and an accuracy of $87.5 \%(63 / 72)$.

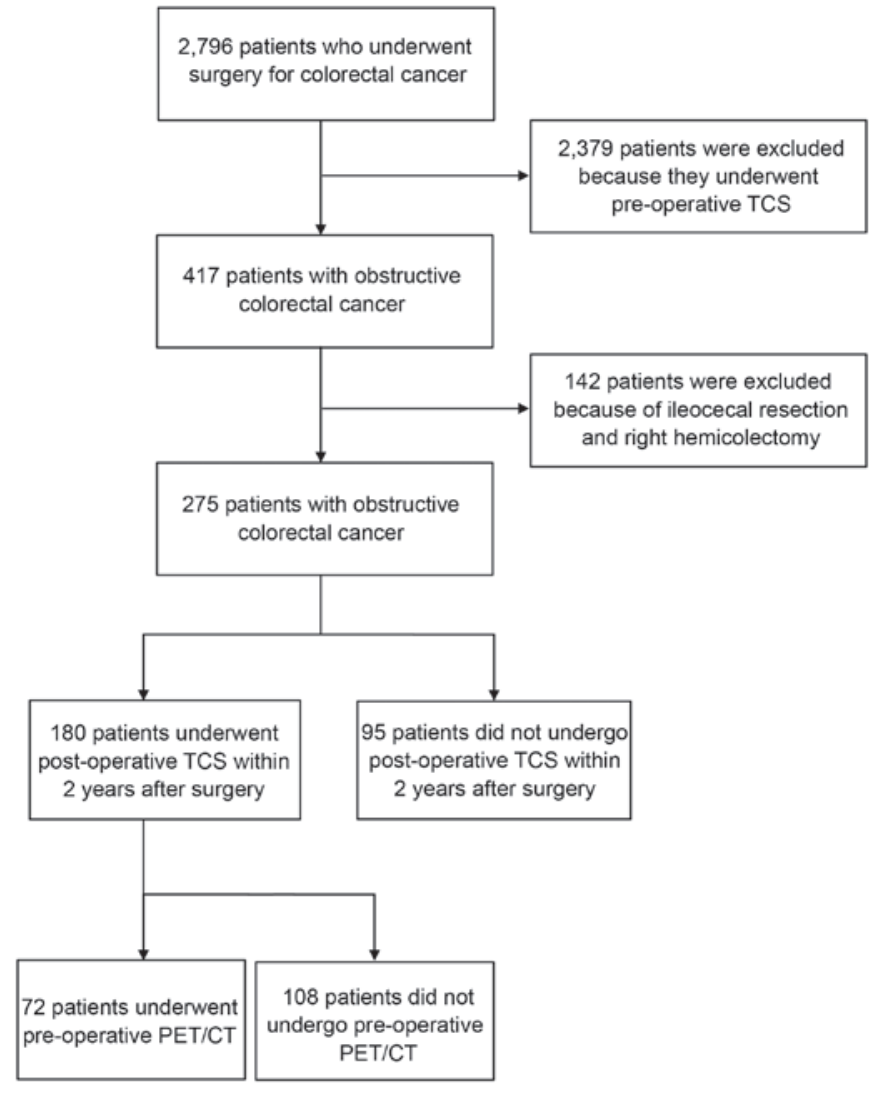

Figure 1. Flowchart for patient selection. TCS, total colonoscopy; PET-CT, positron emission tomography/computed tomography.

Clinical characteristics of the 11 patients with FDG uptake during the PET/CT scan of the oral side of the colon. Eleven patients exhibited FDG uptake in the colon, and these patients had three advanced adenomas (a 20-mm Is mucosal adenoma, a 28-mm IIa mucosal adenoma, and an 18-mm Is mucosal adenoma), four invasive cancers (a 45-mm pT3 tumor, a 22-mm pT2 tumor, a $70-\mathrm{mm}$ pT3 tumor, and a $23-\mathrm{mm}$ pT3 tumor), and four false-positive results (Table II). The patients with the 22-mm pT2, 70-mm pT3, and 23-mm pT3 tumors were treated using a single-stage operation, and the patient with the $45-\mathrm{mm}$ pT3 tumor was treated using a two-stage operation. The first stage involved resection of the rectosigmoid colon cancer, although the patient had severe left ventricular dysfunction and the pT3 tumor in the splenic flexure was not palpated during the surgery. After the cancer in the splenic flexure was diagnosed using postoperative TCS, it was resected during a second surgery. Three patients with an advanced adenoma underwent endoscopic mucosal resection after the original surgery.

One of the four patients with false-positive results exhibited FDG uptake in the terminal ileum, and sigmoidectomy and ileum resection were performed because the ileum was adhered to the primary sigmoid colon cancer. This patient had previously experienced cecum perforation, and the pathological diagnosis was granuloma. The other three patients with false-positive results underwent further evaluation, and no $>5$ - $\mathrm{mm}$ polyps were detected near the site of the FDG uptake.

Clinical strategy for obstructive colorectal cancer. The remaining 61 patients did not exhibit FDG uptake in the 
Table I. Associations between FDG uptake and oral-sided colonic lesions detected using postoperative TCS.

Patient underwent pre-op PET/CT

Colonic lesion detected

Positive findings

Negative findings

No pre-op PET/CT performed

Total

Proximal advanced lesions ${ }^{\mathrm{a}}(+)$

\section{7}

Advanced adenoma ${ }^{\mathrm{b}}$

Invasive cancer

Proximal advanced lesions (-)

Total no. of lesions
3

4

4

11

6
4
2
55
61

13

9

4

95

108

26
16
10
154
180

${ }^{a}$ Advanced lesion: Advanced adenoma and invasive cancer; ${ }^{b}$ Advanced adenoma: Adenomas that were $\geq 10$ mm and mucosal cancers. FDG, fluorodeoxyglucose; TCS, total colonoscopy; PET-CT, positron emission tomography/computed tomography.

Table II. Clinical characteristics of the 11 patients with FDG uptake during the PET/CT scan of the oral side of the colon.

\begin{tabular}{|c|c|c|c|c|c|c|c|c|c|}
\hline \multirow[b]{2}{*}{ Case } & \multirow[b]{2}{*}{ Age/sex } & \multicolumn{3}{|c|}{ Primary colorectal cancer } & \multicolumn{3}{|c|}{ Proximal colorectal lesions } & \multirow[b]{2}{*}{ Intraoperative palpation } & \multirow[b]{2}{*}{ Treatment } \\
\hline & & Location & Depth & Size $(\mathrm{mm})$ & Location/histology & Depth & Size $(\mathrm{mm})$ & & \\
\hline 1 & $73 / \mathrm{M}$ & $\mathrm{RS}$ & $\mathrm{T} 3$ & 60 & $\mathrm{D} /$ cancer & $\mathrm{T} 3$ & 45 & Not palpable & Two-stage op \\
\hline 2 & $85 / \mathrm{M}$ & S & T3 & 42 & S/cancer & $\mathrm{T} 2$ & 22 & Palpable & One-stage op \\
\hline 3 & $75 / \mathrm{M}$ & S & $\mathrm{T} 4$ & 40 & S/cancer & $\mathrm{T} 3$ & 70 & Palpable & One-stage op \\
\hline 4 & $65 / \mathrm{F}$ & $\mathrm{D}$ & $\mathrm{T} 3$ & 50 & $\mathrm{D} /$ cancer & $\mathrm{T} 3$ & 23 & Palpable & One-stage op \\
\hline 5 & $53 / \mathrm{M}$ & $\mathrm{S}$ & $\mathrm{T} 3$ & 52 & T/cancer & Tis & 20 & Palpable & $\begin{array}{l}\text { Op }+ \text { endoscopic } \\
\text { resection }\end{array}$ \\
\hline 6 & $89 / \mathrm{M}$ & $\mathrm{R}$ & $\mathrm{T} 3$ & 95 & $\mathrm{C} /$ cancer & Tis & 28 & Palpable & One-stage op \\
\hline 7 & $61 / \mathrm{M}$ & $\mathrm{Ra}$ & $\mathrm{T} 4$ & 62 & S/cancer & Tis & 18 & Palpable & One-stage op \\
\hline 8 & $63 / \mathrm{M}$ & $\mathrm{RS}$ & $\mathrm{T} 3$ & 61 & $\mathrm{C} /$ granuloma & - & - & Palpable & One-stage op \\
\hline 9 & $70 / \mathrm{M}$ & $\mathrm{Ra}$ & $\mathrm{T} 3$ & 64 & C/hyper & - & 3 & Not palpable & - \\
\hline 10 & $67 / F$ & S & $\mathrm{T} 3$ & 40 & A/hyper & - & 4 & Not palpable & - \\
\hline 11 & $71 / \mathrm{M}$ & S & $\mathrm{T} 3$ & 46 & A & - & - & Not palpable & - \\
\hline
\end{tabular}

FDG, fluorodeoxyglucose; M, male; F, female; C, cecum; A, ascending colon; T, transverse colon; D, descending colon; S, sigmoid colon; RS, rectosigmoid colon; Ra, upper rectum; Hyper, hyperplastic polyp; PET-CT, positron emission tomography/computed tomography; Tis, carcinoma in situ; T2, tumor invades muscularis propria; T3, tumor invades through the muscularis propria into pericolorectal tissues; T4, tumor penetrates the surface of the visceral peritoneum.

proximal colon. Postoperative TCS subsequently revealed four advanced adenomas (a 12-mm IIa adenoma, a 6-mm IIa mucosal cancer, a 20-mm IIa adenoma, and a 20-mm Ip adenoma) and two invasive cancers (a 7-mm Is pT1b tumor and a 12-mm IIa pT1b tumor). One hundred and eight patients did not undergo preoperative PET/CT, and postoperative TCS subsequently revealed nine advanced adenomas and four invasive cancers. There was no significant difference in the incidences of proximal colonic lesions when we compared the groups with and without positive findings during the preoperative PET/CT (Fisher's exact test).

\section{Discussion}

Synchronous colon cancers occur in $2-5 \%$ of patients with colorectal cancer $(5,6)$. Thus, as patients with obstructive cancers cannot be evaluated using preoperative TCS, other methods are needed to assess the proximal colon, such as CT colonography and self-expandable metallic stents. For example, a self-expanding metal stent can be placed endoscopically to facilitate preoperative TCS and provide a bridge to surgery in patients with obstructive tumors, as a Japanese study revealed that it provided a high technical success rate and a low complication rate among patients with colorectal cancer (14). However, the European Society of Gastrointestinal Endoscopy clinical guidelines do not recommend colonic self-expanding metal stent placement as a bridge to elective surgery (15), and we believe it is better to avoid stent use until the long-term oncological and safety outcomes are confirmed. In addition, endoscopic observation of the proximal colon after stenting can cause stent migration or perforation. Therefore, stenting does not always make preoperative TCS possible. In contrast, CT colonography can be used to detect synchronous proximal colon cancers in 
patients with obstructive lesions (16), although the procedure requires adequate bowel preparation, fecal tagging, and colonic distension using air or CO2. Nevertheless, PET/CT can detect colonic lesions even if the colon proximal to the obstruction is not adequately prepared (17).

As PET/CT provides a high detection rate for colorectal cancer, the present study evaluated its ability to detect cancers in patients with obstructive colon cancer. Compared to barium enema or intraoperative colonoscopy, PET is a less invasive screening technique that uses FDG (a commonly used PET tracer), which is a glucose analogue with no risks of side effects or pharmacological allergies. However, PET is associated with several disadvantages, such as FDG uptake being affected by cell density. Therefore, it is difficult for PET to identify malignant tumors with low cell density, such as bronchioloalveolar carcinoma or gastric signet ring cell carcinoma $(18,19)$. Hyperglycemia can also cause false-negative results for FDG uptake in the body (20). Another disadvantage of PET is that its spatial resolution is inferior to that of CT, and an improved diagnostic ability is achieved using the combination of PET and CT (21).

Previous studies have indicated that PET has a high sensitivity (90\%) and specificity (66\%) for identifying primary colorectal tumors (2), and FDG-PET provides a sensitivity of $90 \%$ for detecting metastatic liver lesions, with a specificity of $100 \%$ (1). Another common use of PET is screening for colorectal cancer (22), and Friedland et al (23) have reported that PET detected $100 \%$ of malignant lesions with a diameter of $\geq 2 \mathrm{~cm}$, but only $17 \%$ of lesions with a diameter of $<2 \mathrm{~cm}$, and that PET had lower sensitivity for detecting flat adenomatous lesions than for protruding lesions (23). Another study revealed that $90 \%$ of adenomatous polyps with a diameter of $\geq 13 \mathrm{~mm}$ were detected incidentally during FDG-PET (24). In particular, villous adenomas tend to have elevated FDG uptake (25), and the lesion's size and its morphological or pathological type can affect the ability to identify colorectal lesions. The large intestine is a well-known site of physiological FDG uptake, and intestinal FDG uptake also poses a practical problem during PET, as it can disguise FDG uptake by adenomatous lesions (26).

In the present study, false-negative PET/CT results were observed for four advanced adenomas (a 6-mm IIa mucosal cancer, a 12-mm IIa adenoma, a 20-mm IIa adenoma, and a $20-\mathrm{mm}$ Ip adenoma) and two invasive cancers (a 7-mm Is pT1b tumor and a 12-mm IIa pT1b tumor). These mucosal and invasive cancers were relatively small, and their FDG uptake was likely affected by their grade of differentiation and number of tumor cells. However, the negative predictive value was high for both synchronous advanced lesions (90.2\%, 55/61) and invasive cancers $(96.7 \%, 59 / 61)$, which suggests that negative PET results indicate a low probability of proximal invasive colon cancer. Postoperative endoscopy can be used to treat proximal mucosal cancer or advanced adenoma, which are both movable during intraoperative palpation because they are not firmly adherent to the muscularis propria. Based on these results, we have developed a clinical strategy for treating obstructive colon cancer (Fig. 2). In this strategy, patients with negative PET results can undergo postoperative TCS, while patients with positive PET results should undergo preoperative TCS using

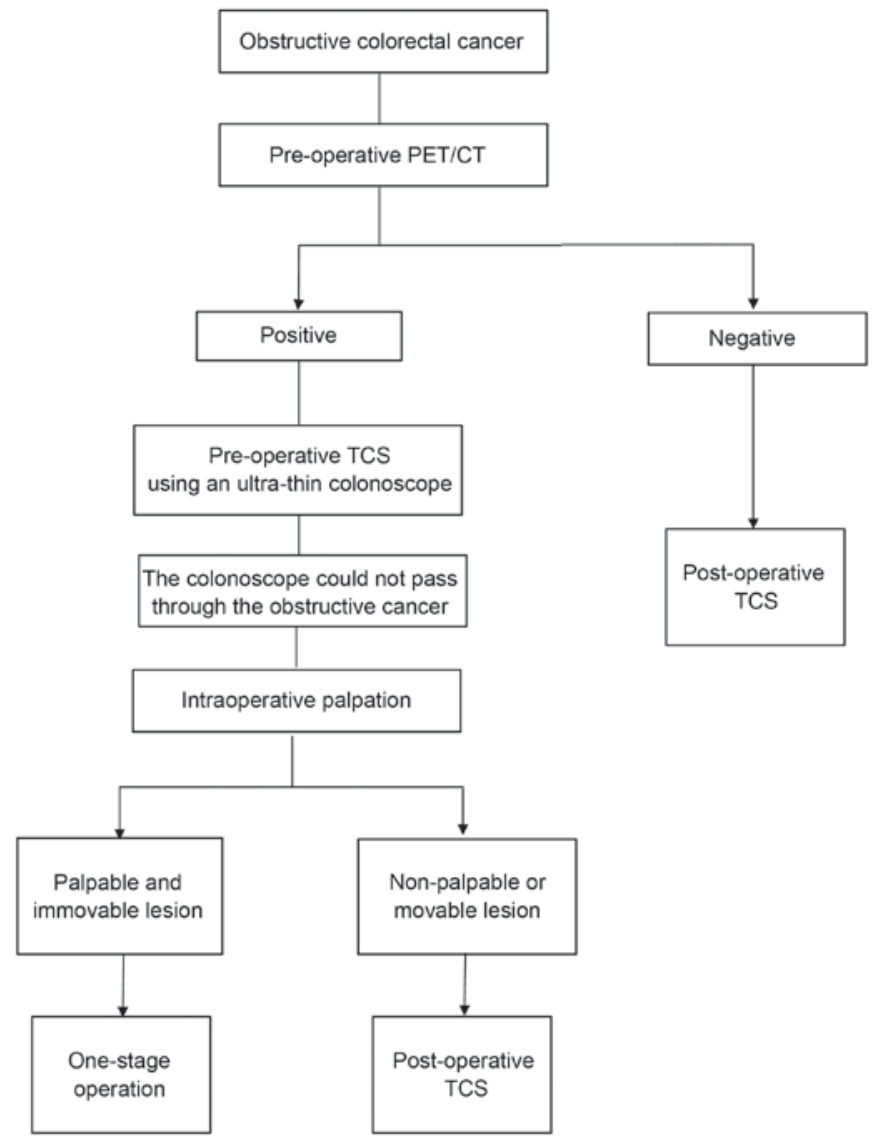

Figure 2. Clinical strategy for obstructive colorectal cancer. TCS, total colonoscopy; PET-CT, positron emission tomography/computed tomography.

an ultra-thin colonoscope. If the ultra-thin colonoscope cannot pass through a cancer-related obstruction, intraoperative palpation is needed. Patients with negative palpation results, or lesions that are movable and palpable, can undergo postoperative TCS. Lesions that have positive PET results and are not movable during palpation may be suitable for a single-stage surgery. As intraoperative palpation is difficult during laparoscopic surgery, it may be necessary to perform hand-assisted laparoscopic surgery to facilitate the palpation. Moreover, we misdiagnosed a case of colon cancer in the splenic flexure, which suggests that splenic flexure uptake during PET/CT requires careful attention during the surgery.

The present study has several limitations. First, the retrospective design is associated with known limitations and risks of bias. Second, the TCS was not performed within 1 year after surgery, which is a requirement for diagnosing synchronous colon lesions. Third, the patients did not undergo colonoscopy using an ultra-thin colonoscope (outer diameter: $6.8 \mathrm{~mm}$ ). Nevertheless, relative to CT colonography and self-expanding metal stent placement, PET/CT is less invasive and more useful for detecting proximal colon cancers.

Based on the high negative predictive value, negative PET/CT results indicate a low probability of synchronous colon cancer in the proximal colon. Therefore, PET/CT may be a useful tool for detecting synchronous colonic cancers in patients with obstructive colon cancer. 


\section{Acknowledgements}

Not applicable.

\section{Funding}

No funding was received.

\section{Availability of data and materials}

All data generated or analyzed during the present study are included in this published article.

\section{Authors' contributions}

CM, SE and SK conceived and designed the present study. CM, SE, YM, SM, EH and FI acquired the data. CM and SE analyzed and interpreted the data. CM, SE and YM wrote and edited the manuscript.

\section{Ethics approval and consent to participate}

The present study's retrospective protocol was approved by the Ethics Committee of Showa University Northern Yokohama Hospital (1310-14; Kanagawa, Japan). Written informed consent was obtained via the opt-out method.

\section{Patient consent for publication}

Not applicable.

\section{Competing interests}

The authors declare that they have no competing interests.

\section{References}

1. Vitola JV, Delbeke D, Sandler MP, Campbell MG, Powers TA, Wright JK, Chapman WC and Pinson CW: Positron emission tomography to stage suspected metastatic colorectal carcinoma to the liver. Am J Surg 171: 21-26, 1996.

2. Thoeni RF: Colorectal cancer. Radiologic staging. Radiol Clin North Am 35: 457-485, 1997.

3. Moertel CG, Bargen JA and Dockerty MB: Multiple carcinomas of the large intestine: A review of the literature and a study of 261 cases. Gastroenterology 34: 85-98, 1958.

4. Berson HL and Berger L: Multiple carcinomas of the large intestine. Surg Gynecol Obstet 80: 75-84, 1945.

5. Passman MA, Pommier RF and Vetto JT: Synchronous colon primaries have the same prognosis as solitary colon cancers. Dis Colon Rectum 39: 329-334, 1996.

6. Ringland CL, Arkenau HT, O'Connell DL and Ward RL: Second primary colorectal cancers (SPCRCs): Experiences from a large Australian Cancer Registry. Ann Oncol 21: 92-97, 2010.

7. Rex DK, Johnson DA, Anderson JC, Schoenfeld PS, Burke CA and Inadomi JM; American College of Gastroenterology: American College of Gastroenterology guidelines for colorectal cancer screening 2009. Am J Gastroenterol 104: 739-750, 2009.

8. Cappell MS and Friedel D: The role of sigmoidoscopy and colonoscopy in the diagnosis and management of lower gastrointestinal disorders: Technique, indications, and contraindications Med Clin North Am 86: 1217-1252, 2002.

9. Desch CE, Benson AB III, Somerfield MR, Flynn PJ, Krause C, Loprinzi CL, Minsky BD, Pfister DG, Virgo KS and Petrelli NJ American Society of Clinical Oncology: Colorectal cancer surveillance: 2005 update of an American Society of Clinical Oncology practice guideline. J Clin Oncol 23: 8512-8519, 2005.
10. Rex DK, Kahi CJ, Levin B, Smith RA, Bond JH, Brooks D, Burt RW, Byers T, Fletcher RH, Hyman N, et al; American Cancer Society; US Multi-Society Task Force on Colorectal Cancer: Guidelines for colonoscopy surveillance after cancer resection: A consensus update by the American Cancer Society and the US Multi-Society Task Force on Colorectal Cancer. Gastroenterology 130: 1865-1871, 2006.

11. Fenlon HM, McAneny DB, Nunes DP, Clarke PD and Ferrucci JT: Occlusive colon carcinoma: Virtual colonoscopy in the preoperative evaluation of the proximal colon. Radiology 210: 423-428, 1999.

12. Chen HS and Sheen-Chen SM: Synchronous and 'early' metachronous colorectal adenocarcinoma: Analysis of prognosis and current trends. Dis Colon Rectum 43: 1093-1099, 2000.

13. Mori S and Oguchi K: Application of (18)F-fluorodeoxyglucose positron emission tomography to detection of proximal lesions of obstructive colorectal cancer. Jpn J Radiol 28: 584-590, 2010.

14. Saida Y, Enomoto T, Takabayashi K, Otsuji A, Nakamura Y, Nagao J and Kusachi S: Outcome of 141 cases of self-expandable metallic stent placements for malignant and benign colorectal strictures in a single center. Surg Endosc 25: 1748-1752, 2011

15. van Hooft JE, van Halsema EE, Vanbiervliet G, Beets-Tan RG, DeWitt JM, Donnellan F, Dumonceau JM, Glynne-Jones RG, Hassan C, Jiménez-Perez J, et al; European Society of Gastrointestinal Endoscopy: Self-expandable metal stents for obstructing colonic and extracolonic cancer: European Society of Gastrointestinal Endoscopy (ESGE) Clinical Guideline. Endoscopy 46: 990-1053, 2014.

16. Nagata K, Endo S, Tatsukawa K and Kudo SE: Double colorectal cancer only diagnosed by computed tomographic colonography. Case Rep Gastroenterol 2: 44-48, 2008.

17. Nagata K, Ota Y, Okawa T, Endo S and Kudo SE: PET/CT colonography for the preoperative evaluation of the colon proximal to the obstructive colorectal cancer. Dis Colon Rectum 51: 882-890, 2008.

18. Higashi K, Ueda Y, Seki H, Yuasa K, Oguchi M, Noguchi T, Taniguchi $\mathrm{M}$, Tonami $\mathrm{H}$, Okimura $\mathrm{T}$ and Yamamoto I: Fluorine-18-FDG PET imaging is negative in bronchioloalveolar lung carcinoma. J Nucl Med 39: 1016-1020, 1998.

19. Stahl A, Ott K, Weber WA, Becker K, Link T, Siewert JR, Schwaiger $M$ and Fink U: FDG PET imaging of locally advanced gastric carcinomas: Correlation with endoscopic and histopathological findings. Eur J Nucl Med Mol Imaging 30: 288-295, 2003.

20. Kubota K: From tumor biology to clinical Pet: A review of positron emission tomography (PET) in oncology. Ann Nucl Med 15: 471-486, 2001.

21. Cohade C, Osman M, Leal J and Wahl RL: Direct comparison of (18)F-FDG PET and PET/CT in patients with colorectal carcinoma. J Nucl Med 44: 1797-1803, 2003.

22. Minamimoto R, Senda M, Uno K, Jinnouchi S, Iinuma T, Ito K, Okuyama C, Oguchi K, Kawamoto M, Suzuki Y, et al: Performance profile of FDG-PET and PET/CT for cancer screening on the basis of a Japanese Nationwide Survey. Ann Nucl Med 21: 481-498, 2007.

23. Friedland S, Soetikno R, Carlisle M, Taur A, Kaltenbach T and Segall G: 18-Fluorodeoxyglucose positron emission tomography has limited sensitivity for colonic adenoma and early stage colon cancer. Gastrointest Endosc 61: 395-400, 2005.

24. Yasuda S, Ide M, Fujii H, Nakahara T, Mochizuki Y, Takahashi W and Shohtsu A: Application of positron emission tomography imaging to cancer screening. Br J Cancer 83: 1607-1611, 2000.

25. Arslan N, Dehdashti F and Siegel BA: FDG uptake in colonic villous adenomas. Ann Nucl Med 19: 331-334, 2005.

26. Yasuda S, Fujii H, Nakahara T, Nishiumi N, Takahashi W, Ide M and Shohtsu A: 18F-FDG PET detection of colonic adenomas. J Nucl Med 42: 989-992, 2001.

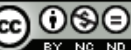

This work is licensed under a Creative Commons Attribution-NonCommercial-NoDerivatives 4.0 International (CC BY-NC-ND 4.0) License. 